\title{
'I don't know why they don't come': barriers to participation in cardiac rehabilitation
}

Michelle L. DiGiacomo ${ }^{1,8}$ BA, MHSc(Hons), PhD, Postdoctoral Research Fellow

Sandra C. Thompson ${ }^{2,3}$ BSc (Med)(Hons), MBBS(Hons), GradDipHlthMgt, MPH, PhD, FAFPHM, Professor

Julie S. Smith ${ }^{4,5}$ RN, BSc, Secondary Prevention Project Officer, Clinical Nurse Specialist

Kate P. Taylor ${ }^{3}$ BA, MIH, Aboriginal Health Research and Professional Education Officer

Lynette A. Dimer ${ }^{4}$ EN, Aboriginal Health Coordinator

Mohammed A. Ali ${ }^{3}$ MBBS, PGDipPHC, MMedSc, Lecturer

Marianne M. Wood 6 MBChB, DPH, BA, General Practitioner

Timothy G. Leahy ${ }^{7}$ MBBS, MFM, FRACGP, Public Health Medical Officer

Patricia M. Davidson ${ }^{1}$ RN, BA, MEd, PhD, Professor

${ }^{1}$ Centre for Cardiovascular and Chronic Care, Curtin Health Innovation Research Institute, Curtin University and University of Technology Sydney, Level 7, Building 10, 235-253 Jones Street, Ultimo NSW 2007, Australia. Email: p.davidson@curtin.edu.au

${ }^{2}$ Combined Universities Centre for Rural Health, University of Western Australia, PO Box 109, Geraldton, WA 6531, Australia. Email: sandra.thompson@cucrh.uwa.edu.au

${ }^{3}$ Centre for International Health, Curtin Health Innovation Research Institute, Curtin University, GPO Box U1987, Perth, WA 6845, Australia. Email: k.taylor@curtin.edu.au; m.ali@curtin.edu.au

${ }^{4}$ National Heart Foundation (Western Australia), 334 Rokeby Road, Subiaco, WA 6008, Australia.Email: julie.smith@heartfoundation.com.au; lynette.dimer@heartfoundation.com.au ${ }^{5}$ Royal Perth Hospital, GPO Box X2213, Perth, WA 6001, Australia.

6 Derbarl Yerrigan Health Service, 156 Wittenoom Street, East Perth, WA 6004, Australia. Email: woodm@derbarlyerrigan.com.au

${ }^{7}$ Aboriginal Health Council of Western Australia, Dilhorn House, 2 Bulwer Street, Perth, WA 6849, Australia. Email: tim.leahy@ahcwa.org

${ }^{8}$ Corresponding author. Email: m.digiacomo@curtin.edu.au 


\section{Abstract \\ Objectives}

To describe health professionals’ perceptions of Aboriginal people’s access to cardiac rehabilitation (CR) services and the role of institutional barriers in implementing the National Health and Medical Research Council (NHMRC) guidelines Strengthening Cardiac Rehabilitation and Secondary Prevention for Aboriginal and Torres Strait Islander peoples.

\section{Design}

Qualitative study

\section{Setting}

Metropolitan and rural tertiary and community-based public CR services and Aboriginal health services in WA.

\section{Participants}

Thirty-eight health professionals working in the CR setting.

\section{Method}

Semi-structured interviews were undertaken with 28 health professionals at public CR services and 10 health professionals from Aboriginal Medical Services in WA. The participants represented 17 services (10 rural, 7 metropolitan) listed in the WA Directory of CR services.

\section{Results}

Emergent themes included (1) a lack of awareness of Aboriginal CR patients' needs; (2) needs related to cultural awareness training for health professionals; and (3) Aboriginal health staff facilitate access for Aboriginal patients.

\section{Conclusions}

Understanding the institutional barriers to Aboriginal participation in CR is necessary to recommend viable solutions. Promoting cultural awareness training, recruiting Aboriginal health workers and monitoring participation rates are important in improving health outcomes. 


\section{What is already known about this subject?}

Significant health and social inequity exists for Aboriginal Australians. Despite the persisting high rates of morbidity and mortality related to cardiovascular disease in Aboriginal Australians, participation rates in cardiac rehabilitation remain low.

\section{What does this paper add?}

Despite widespread dissemination of NHMRC guidelines, there remains a disconnect between CR health professionals’ understandings and practices and the needs of Aboriginal people in WA. Increasing the volume and quality of cultural awareness training as well as access to Aboriginal health professionals are crucial in addressing this disparity.

\section{What are the implications for practitioners?}

Increasing the number and support of Aboriginal people trained as health professionals will assist the system to respond better to the needs of communities. Collaborative partnership models where Aboriginal and non-Aboriginal health professionals work together to increase mutual understanding are warranted. 


\section{Introduction}

The health disparities of Indigenous people around the world are a global public health issue. In Australia, Aboriginal and Torres Strait Islander peoples are more likely to have cardiovascular disease and suffer more fatal coronary events than other Australians. ${ }^{1}$ Cardiac rehabilitation (CR) is a structured approach to secondary prevention and cardiac care involving exercise, behavioural modification and education, and is endorsed by Australian state and federal government policy. ${ }^{2}$ Following an acute coronary event, CR has proven effective in reducing cardiovascular risk. Although participation rates in CR are less than optimal for all Australians, Aboriginal participation in CR programs is significantly lower. ${ }^{3}$ The barriers to service access for Aboriginal patients and developing enabling strategies are critical public health issues. In spite of targeted guidelines to promote access, uptake and adherence is limited. ${ }^{4}$

In 2008, Australian Prime Minister Kevin Rudd offered a historic apology to Aboriginal people and the Stolen Generations, in which he emphasised the importance of improving health services for Aboriginal people and engaging Aboriginal people in health research, services, and education to reduce health inequities, improve access, and achieve equitable policy out- comes. ${ }^{5}$ Engaging consumers in the planning, delivery and evaluation of health care arises from the belief that better health outcomes result from collaborative, community-involved decision-making. ${ }^{6}$ However, ensuring that collaboration with Ab- original health service consumers genuinely results in service translation can be challenged by a lack of understanding of cultural differences. ${ }^{7}$ Overcoming these challenges requires appreciating the beliefs and values of an individual's culture and adopting approaches that facilitate appropriate patient-provider interactions. This process, known as cultural competence in health care, appreciates the social and cultural influences on patients' health beliefs and behaviours and adopts steps to incorporate these understandings into patient-provider interactions and, ultimately, service delivery. ${ }^{8}$ 
In 2005, the National Health and Medical Research Council (NMHRC) released Strengthening Cardiac Rehabilitation and Secondary Prevention for Aboriginal and Torres Strait Islander Peoples: A Guide for Health Professionals ${ }^{9}$ (hereafter, the Guide). The Guide provides health professionals with a practical frame- work for delivering culturally competent and improved CR services to Aboriginal people. These guidelines provide information on cultural competency and approaches to improving Aboriginal participation in CR as well as checklists for health professionals working in primary care, CR, and health management settings. The Guide was distributed to a wide range of interested and relevant individuals and organisations, including Aboriginal Community Controlled Health Services, throughout Australia. Limited implementation strategies have shown inadequate awareness of these guidelines. ${ }^{4}$

In Western Australia (WA), there is disparity in the health and life expectancy between Aboriginal and non-Aboriginal Australians. ${ }^{10}$ The remoteness of many areas and unbalanced population distribution combined with cultural differences contribute to complexities in health service planning and effective delivery for Aboriginal communities. ${ }^{6}$ To better understand CR services available to Aboriginal people in WA, a project was undertaken to assess CR implementation of the Guide statewide. The Guide included a seven-page toolkit on cultural competency, including avenues to obtain further information and develop cultural expertise, tips on communicating with individuals, communities, and Aboriginal Health Services, using appropriate language, working with cultural mentors, interpreters, and tips for providing organisational support for intercultural service delivery.

As part of the initial evaluation, a striking finding, particularly within non-Aboriginal-specific health facility participants, was their bemusement regarding why Aboriginal people do not attend CR. This lack of awareness between health professionals and the circumstances of their patients ${ }^{11}$ is not confined to 
relationships between Aboriginal and non-Aboriginal people, but represents an additional layer of complexity. Building upon a previous report, this paper presents findings on health professionals' perceptions of Aboriginal patients’ access to secondary prevention services and avenues for increasing awareness, namely via cultural aware- ness training and the Aboriginal Health workforce, both of which are espoused within the Guide.

\section{Methods}

The sampling frame comprised health service organisations included in the Directory of Western Australian Secondary Prevention Services ${ }^{12}$ and Aboriginal Medical Services in WA. A CR nurse contacted services to discuss the study and arranged a site visit for an interview upon which formal written consent was obtained. Private health facilities who serviced few Indigenous clients or who had few or no CR patients or referrals were excluded. Participants were interviewed between November 2007 and March 2008 by a CR nurse accompanied, when possible, by an Aboriginal nurse. This dyad fostered rapport building during interviews and aimed to ensure an Aboriginal perspective to the evaluation of CR programs and cultural safety within the service. Ethical approval was obtained from the WA Aboriginal Health Information and Ethics Committee and the Curtin University Human Research Ethics Committee.

Semi-structured interviews were conducted with health professionals at their organisations regarding their awareness, implementation, and perspectives of barriers to implementation of the Guidelines. Of particular interest were their perceptions of CR services provided for Aboriginal people, the capacity of programs and personnel to meet needs of local Aboriginal communities, and availability of cultural awareness training. Interview topics are listed in Box 1. Written notes were taken during interviews with three participants who declined audio-recording whereas all other interviews were audio-recorded and transcribed verbatim. Thematic content analysis of transcripts involved open coding, in which the data 
were broken down into distinct units of meaning, or codes, according to participants' responses. ${ }^{13}$ Codes were repeatedly compared with one another and links between cate- gories were established. ${ }^{13}$ Related categories were combined, compared with new data, and further developed. Steps were undertaken to maximise reflexivity and rigour through discussion within the research team and verification and clarification of themes emerging from the data. ${ }^{14}$

\section{Box 1. Interview topics}

* Awareness of, and perceived barriers to, implementation of NHMRC Guidelines

* Type, structure, content, organisation, coordination, staffing and funding of the service with specific relevance to Aboriginal people

* Aboriginal Health Worker involvement in care of Aboriginal patients

* Collaboration and integration with existing service providers

* Cultural awareness training

\section{Results}

Health professionals from 17 of the 31 organisations comprising the Directory of Western Australian Secondary Prevention Services ${ }^{15}$ and seven Aboriginal health service organisations participated in the interviews. In total, 38 semi-structured interviews were conducted with staff from public CR services (28) and Aboriginal health and medical services (10) in WA. Themes that emerged from the data were (1) lack of awareness of Aboriginal CR patients' needs; (2) needs related to cultural awareness training for health professionals; and (3) Aboriginal health staff facilitate access for Aboriginal patients. Verbatim quotes sup- porting these themes are provided in Table 1.

\section{Lack of awareness of Aboriginal CR patients’ needs}

One theme that emerged from the interviews was an apparent disconnection between CR services and Aboriginal clients, reflected by poor participation rates in programs. Although several participants conveyed an awareness of needs and appreciated the depth and complexity of issues facing Aboriginal patients, some participants employed at government-funded health services and hospitals 
expressed a lack of understanding of Aboriginal patients’ experiences and needs. In particular, some respondents were unfamiliar with barriers that Aboriginal people faced in regard to accessing CR.

Although some respondents were aware of aspects of CR services not conducive to Aboriginal cultural norms and behaviours, others remained puzzled by low attendance rates, highlighting the need for increased cultural awareness and information about strategies to promote engagement. Few respondents reported community involvement in the design and delivery of CR program initiatives and many services were also unaware that there were CR support resources available for regional settings. A concern for some respondents was that metropolitan-based health professionals, particularly in hospitals, appeared not to understand the extent that information and strategies for disease management needed to be tailored for Aboriginal patients who were returning back to regional settings. For example, environmental issues, such as lack of dedicated exercise facilities (pools and gyms) and safety issues when exercising outdoors (such as excessive heat or rain, flies, glass on footpaths, and roaming dogs) can create barriers for Aboriginal patients in engaging in CR activities. Perspectives regarding avenues for increasing health professionals’ awareness of Ab- original patients’ experiences involved cultural awareness training and engagement with the Aboriginal Health workforce.

\section{Perspectives of cultural awareness training within health services}

Staff in hospitals or state-based facilities reported more often that their organisations did not offer, or that they were not aware of, cultural awareness training programs. Some participants explained that within these services, staff cultural awareness training was part of new staff orientation, although sometimes this process occurred almost a year into employment or was considered brief. Participants who were recently migrated health professionals and those practicing in remote areas spoke of the increased relevance for their cultural awareness training. Engaging continuing staff in cultural awareness training was sometimes challenging due to staff workloads, time constraints, the need to travel vast distances, perceptions of cost-ineffectiveness, and perceptions of non-useful program content. In light of these barriers, neither health professionals, nor their employing institutions, in many cases, 
prioritised cultural awareness training.

In contrast, most staff from Aboriginal health and medical services had access to cultural awareness training and all looked to their Aboriginal Health Worker (AHW) colleagues for cultural mentorship. They expressed the need to institute more expansive cultural awareness programs in non-Aboriginalspecific health services for the good of staff and Aboriginal patients. Despite low uptake and regard for cultural awareness training reported by some respondents, others provided examples of exemplary programs and resources. One Aboriginal Medical Service (AMS) has a program where new staff, including doctors, tour and stay the night in a local community serviced by the AMS, while mentored by an AHW. Another respondent from a main- stream health service explained that their organisation’s program had community-dwelling Aboriginal people come to the hospital to share their stories and experiences and teach health professionals their ways and beliefs.

\section{Aboriginal health staff as facilitating access to health services}

Participants acknowledged that AHWs are a vital component to health initiatives in that they can break down barriers to accessing health services for Aboriginal people and promote cultural awareness in fellow health professionals. They recognised that AHWs are critical to the health and wellbeing of Aboriginal communities, are pivotal in engaging and maintaining relation- ships with patients and their families, providing culturally appropriate information regarding their care, and facilitate interactions between non-Aboriginal health professionals and patients. It was explained that without availability of cultural awareness training from health services, AHWs are relied upon to teach and support nonIndigenous colleagues. Despite their importance, the majority of non-AMS participants felt that their services did not have sufficient access to Aboriginal staff to deliver appropriate care and advocacy for Aboriginal patients. There was an expressed need for increasing numbers of Aboriginal health professionals in hospitals and for the health care institutions to support them. 


\section{Discussion}

These results indicate that there is a considerable need for investment of health care organisations in cultural awareness training and assessment. This is particularly pertinent in the context of a global health workforce where many newcomers to Australia do not have a keen appreciation of the factors impacting on Aboriginal health. ${ }^{16}$ Importantly, results from this study indicate that aspects of both outreach and in-reach cultural awareness programs are most successful in engendering cultural awareness in health professionals.

However, although cultural awareness training is critical for non-Aboriginal staff, real improvements in community engagement will most effectively be achieved by increasing the Aboriginal health workforce. Increasing the numbers of Aboriginal health professionals also has important implications for health spending. In 2004-05 it was estimated that for every dollar spent on nonAboriginal Australians, $\$ 1.17$ was spent on Aboriginal Australians, ${ }^{17}$ reflecting a mismatch to the disease burden (which is 2.5 times higher in Aboriginal compared with non-Aboriginal Australians). One way to improve resource spending is to increase the numbers of Aboriginal people trained as health professionals as they will assist the system to respond better to the needs of communities. Although such recommendations have been made in many reports over a long period, there has been little effective action by government and health planners. ${ }^{18}$ Recent workforce initiatives by the Council of Australian Governments, at last, show evidence of longer-term planning,

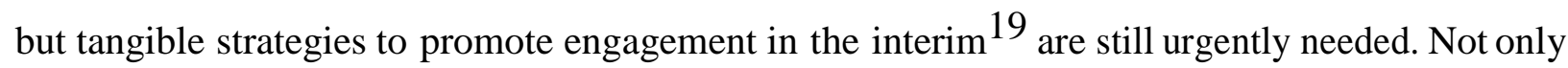
should institutions improve on the numbers of AHWs in hospitals, but institutions need to support them as well. The underemployment and under-utilisation of AHWs in hospitals reflects an institutional barrier; however, it is important for health professionals to be aware of such barriers and plan for or around them where possible. 
Table 1. Quotations from interviews with health professionals

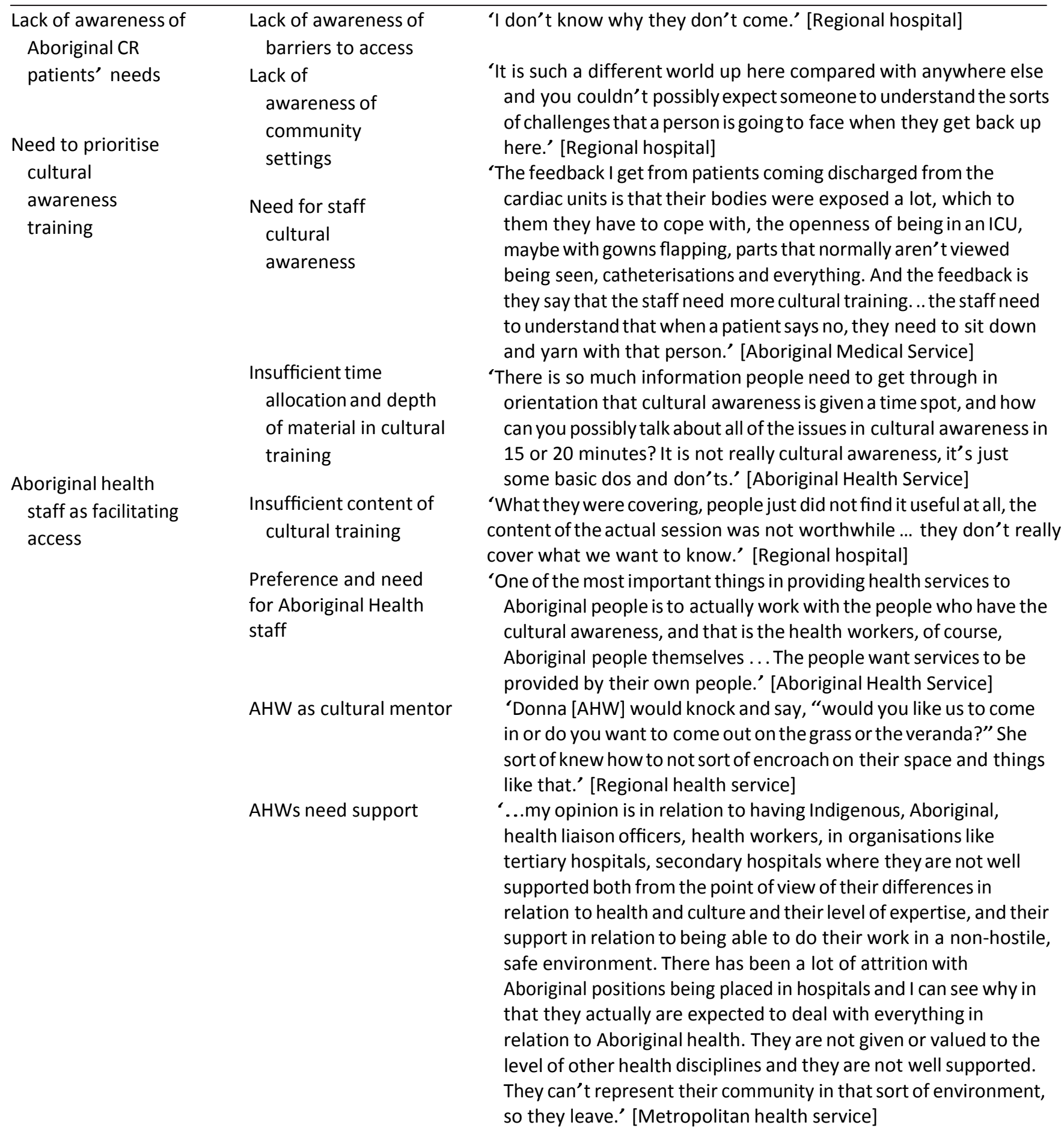


In not addressing these workforce and training issues, government health institutions perpetuate the disconnect between health services and Aboriginal patients. In order to make a real impact on Aboriginal participation, health services need to shift from tokenism to appropriate resourcing and monitoring of outcomes. More resources need to be placed in recruiting Aboriginal health professionals and supporting them to work in the health system.

An important theme emerging from this study is the value of collaborative partnership models where Aboriginal and non-Aboriginal health professionals work together. Other studies have demonstrated how such partnerships can build appreciation of issues impacting on access and forming personal relationships that are critical in community engagement. Poor participation in health service programs by Aboriginal clients undoubtedly contributes to the inferior health outcomes experienced by Aboriginal people. Increasing the engagement of Aboriginal Australians at both consumer participation and a health provider level are critical in terms of improving the capacity of programs to effectively service Aboriginal clients. ${ }^{20}$ Effective data collection systems to monitor CR participation rates for Aboriginal people and increasing access to culturally appropriate models are necessary.

\section{Conclusion}

Despite the release of the Guide in 2005, lack of service knowledge about the resource across WA highlights the need for more effective dissemination and education of service support materials. Many of the issues faced in services in terms of improving Aboriginal participation could be improved if service providers were comprehensively educated about the resource and how to use it in a practical way. Importantly, as indicated by participants, ineffective resource provision and planning can leave obstacles that, if not properly accounted for, can render many of the Guide’s suggestions difficult to implement. Improving the health outcomes of Aboriginal Australians is a complex and multifaceted challenge. 
resources, including both Aboriginal and non- Aboriginal health workers, are crucial in increasing the capacity of the health system to deal with the burden of chronic conditions in Aboriginal people. Increasing the volume and quality of cultural awareness training as well as access to Aboriginal health professionals is crucial in addressing the issue of access to care for Aboriginal people.

\section{Competing Interests}

Authors declare that no conflicts of interest exist.

\section{Acknowledgements}

We thank the participants and health services for their time and gratefully acknowledge colleagues Ms Francine Eades, Dr Tom Briffa, Ms Narelle Wilson and Mr Trevor Shilton for their assistance with this project. We acknowledge the assistance of Royal Perth Hospital and the National Heart Foundation WA. This research was supported by the WA Department of Health through a State Health Research Advisory Council grant and an NHMRC project grant (479222). Dr DiGiacomo is a postdoctoral fellow supported by an NHMRC Capacity Building Grant (533547). 


\section{References}

1 The health and welfare of Australia’s Aboriginal and Torres Strait Islander peoples. Canberra: Australian Bureau of Statistics and Australian Institute of Health and Welfare; 2003.

2 Strengthening Cardiac Rehabilitation and Secondary Prevention for Aboriginal and Torres Strait Islander Peoples: A Guide for Health Professionals. Canberra: Australian Government; 2005.

3 Scott IA, Lindsay K, Hardam H. Utilisation of outpatient cardiac rehabilitation in Queensland. Med J Aust 2003; 179: 341-5.

4 Thompson SC, DiGiacomo ML, Smith J, Taylor K, Dimer L, Ali M, et al. Are the processes recommended by the NHMRC for improving cardiac rehabilitation for Aboriginal and Torres Strait Islander people being implemented? An assessment of CR services across Western Australia. Aust New Zealand Health Policy 2009; 6: 29. doi:10.1186/1743-8462-6-29

5 Alfred A, Kalucy E, McIntyre E. Presenting Australian primary health care research. Adelaide: Primary Health Care Research and Information Service; 2008.

6 Western Australia Health Consumer, Carer and Community Engagement Framework. Perth: Western Australia Department of Health; 2007.

7 Stone L. Cultural influences in community participation in health. Soc Sci Med 1992; 35: 409-17. doi:10.1016/0277-9536(92)90333-L

8 Betancourt JR, Green AR, Carrillo JE, Ananeh-Firempong O. Defining cultural competence: a practical framework for addressing racial/ethnic disparities in health and health care. Public Health Rep 2003; 118:293-302.

9 Strengthening cardiac rehabilitation and secondary prevention for Aboriginal and Torres Strait Islander peoples: a guide for health professionals. Canberra: National Health and Medical Research Council; 2005.

10 Overcoming indigenous advantage in Western Australia. Report 2005.Perth: Department of Indigenous Affairs; 2005.

11 Everett B, Salamonson Y, Zecchin R, Davidson PM. Reframing the dilemma of poor attendance at cardiac rehabilitation: an exploration of ambivalence and the decisional balance. J Clin Nurs 2009; 18(13):1842-9.

12 National Heart Foundation of Australia (WA Division). Directory of Western Australian Secondary 
Prevention Services. Perth: Heart Foundation of WA; 2007.

13 Grbich C. Qualitative Research in Health: An Introduction. Sydney: Allen \& Unwin; 1999.

14 Kitto SC, Chesters J, Grbich C. Quality in qualitative research. Med J Aust 2008; 188(4): $243-6$.

15 National Heart Foundation of Australia. Directory of Western Australian Secondary Prevention Services. Perth: National Heart Foundation of Australia (WA Division); 2007.

16 Gilles MT, Wakerman J, Durey A. 'If it wasn’t for OTDs, there would be no AMS': overseas-trained doctors working in rural and remote Aboriginal health settings. Aust Health Rev 2008; 32(4): 655-63. doi:10.1071/ AH080655

17 Deeble J, Agar J, Goss J. Expenditures on health for Aboriginal and Torres Strait Islander Peoples 200405. Canberra: Australian Institute of Health and Welfare; 2008. Health and Welfare Expenditure series no. 33. Cat. no. HWE 40.

18 Cheng MH. Aboriginal workers key to indigenous health in Australia. Lancet 2007; 370(9598): $1533-6$. doi:10.1016/S0140-6736(07)61648-1

19 Shepherd F, Battye K, Chalmers E. Improving access to cardiac rehabilitation for remote Indigenous clients. Aust N Z J Public Health 2003;27: 632-6. doi:10.1111/j.1467-842X.2003.tb00611.x

20 Davidson PM, DiGiacomo M, Abbott P, Sheerin N, Heal PE, Meine L, et al. A partnership model in the development and implementation of a collaborative, cardiovascular education program for Aboriginal Health Worker. Aust Health Rev 2008; 32(1): 139-46. doi:10.1071/AH080139 